\title{
A Robust Controller For Micro-Sized Agents: The Prescribed Performance Approach
}

\author{
Alper Denasi ${ }^{1}$ and Sarthak Misra ${ }^{1,2}$ \\ ${ }^{1}$ Surgical Robotics Laboratory, Department of Biomechanical Engineering, MIRA-Institute for Biomedical Technology \\ and Technical Medicine, University of Twente, The Netherlands \\ ${ }^{2}$ Department of Biomedical Engineering, University of Groningen and University Medical Centre Groningen, The Netherlands \\ Email: \{a.denasi,s.misra\}@utwente.nl
}

\begin{abstract}
Applications such as micromanipulation and minimally invasive surgery can be performed using micro-sized agents. For instance, drug-loaded magnetic micro-/nano- particles can enable targeted drug delivery. Their precise manipulation can be assured using a robust motion controller. In this paper, we design a closed-loop controller-observer pair for regulating the position of microagents. The prescribed performance technique is applied to control the microagents to follow desired motion trajectories. The position of the microagents are obtained using microscopic images and image processing. The velocities of the microagents are obtained using an iterative learning observer. The algorithm is tested experimentally on spherical magnetic microparticles that have an average diameter of $100 \mu \mathrm{m}$. The steady-state errors obtained by the algorithm are $20 \mu \mathrm{m}$. The errors converge to the steady-state in approximately 8 seconds.
\end{abstract}

\section{INTRODUCTION}

The developments in microfabrication technologies in the last decade allowed the progress of microrobotics research on applications such as minimally invasive surgery, microassembly and environmental remediation [1], [2]. Among these applications, there is a growing demand especially for lessinvasive and non-surgical medical interventions which aims at reducing patient trauma and hospitalization time. Various micro-sized agents such as sperm-driven Micro-Bio-Robots [3], MagnetoSperm [4] and magnetic micro-/nano- particles [5] can be utilized for minimally invasive surgical procedures in the human body. For instance, precise manipulation of magnetic micro-/nano- particles which are loaded with drugs can enable targeted drug delivery, since they can be powered and steered wirelessly by external magnetic field gradients.

The success of microrobots in minimally invasive surgery can be assured by three aspects: (i) a propulsion mechanism which can provide enough power to tackle uncertainties and environmental disturbances; (ii) a real-time imaging modality with adequate resolution; (iii) a robust and accurate motion control system. This paper deals with the design of a robust and accurate motion control system for micro-sized agents. Magnetic microparticles with an average diameter of $100 \mu \mathrm{m}$ are selected for this purpose, since they can be controlled wirelessly using external magnetic field gradients.

Microrobots were controlled initially in open-loop, by means of pulling with magnetic field gradients [6], [7]. These works assumed that the magnetic field direction did not vary

This project (ROBOTAR) has received funding from the European Research Council (ERC) under the European Union's Horizon 2020 Research and Innovation programme (Grant Agreement \#638428).

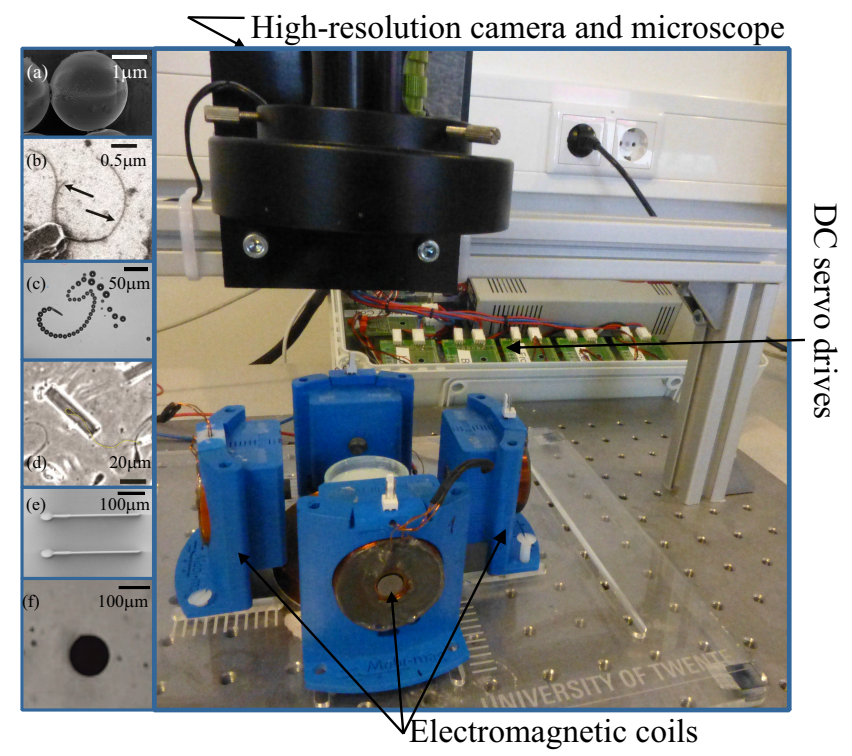

Fig. 1. The Mobi-Mag electromagnetic system used for controlling different type of magnetic microagents using microscope and camera feedback: (a) Janus micromotors (b) Magnetotactic bacteria (c) Self-propelled microjets (d) Sperm-driven micro-bio-robots (e) MagnetoSperm, and (f) Magnetic microparticles [3]-[5]. In order to autonomously move towards a target or follow a desired reference trajectory, these magnetic micro-sized agents need a control algorithm which uses their position information and generates corresponding currents. In this work, we use a prescribed performance controller for this purpose and focus on magnetic microparticles. The electromagnetic coils are used to transmit power wirelessly to the microagents, and the microscope and camera are used to capture images, as shown above. Based on the error between the desired target or trajectory and the actual position, the control signals are generated. These control signals are sent to the DC servo drives shown above which then generate the currents for the electromagnetic coils.

rapidly and neglected the dynamics of the microrobot and the environment. Such assumptions are not valid to perform the previously mentioned applications due to the (possibly nonlinear) perturbations resulting from the environment dynamics, e.g. variations in temperature and viscosity; parametric uncertainties in the magnetic dipole moment [8]. Open-loop control is not suitable to deal with (or rather reject) disturbances. On the contrary, closed-loop control can deal with these perturbations effectively. For microrobotic systems, different closed-loop techniques such as linear control [5], adaptive control [9], $\mathcal{H}_{\infty}$ control [10] and model predictive control [11] have been investigated. For (standard) linear controllers, problems such as instabilities and undesired oscillations have been reported [12], [13]. As a remedy to problems associated with linear controllers, adaptive control has been proposed to estimate drag and electrostatic forces which result from 
the environment dynamics [9]. Since, the drag force is a nonlinear and time-varying function of the viscosity, it cannot be estimated using the classical adaptive approaches [9]. Besides their influence on the closed-loop stability, perturbations degrade the transient and steady-state error performance of the aforementioned controllers. The previously mentioned controllers cannot guarantee the desired transient and steadystate errors without an explicit knowledge of the uncertainties or their upper bounds, which is practically impossible.

A technique which can resolve the previously mentioned stability issues while guaranteeing the desired performance metrics is known as Prescribed Performance Control [14]. Here, prescribed performance refers to the convergence of the control error to a predefined arbitrarily small residual set with a prespecified minimum convergence rate and maximum overshoot level. This technique has been applied to the robust control of manipulators [15] and underwater vehicles [16]. A benefit of this method is due to the fact that contrary to the standard robust and adaptive control methods the output performance is isolated from the control gains selection. Consequently, the robustness against model uncertainties is greatly extended. In [17] the technique is extended with a high gain observer to deal with the absence of the measurement of the full state, i.e., velocities besides the position measurement. State observers such as high gain observers, have also been used in the control of microrobotic systems to estimate the velocities [9]. However, they require an estimation of the dynamics and the knowledge of the control inputs, which depends on the knowledge of the magnetic field gradients. High observer gains are often used to deal with the insufficient knowledge of these variables. Consequently, the noise on the estimated velocities is amplified. The adaptation of the Iterative Learning Observer (ILO) method [18] introduced in [19] does not depend on these variables and thus can deal with the high gain issue.

In this work, we designed a prescribed performance closedloop position controller for microagents which can guarantee the prespecified transient and steady-state error metrics. We obtain the position of the microagents with respect to an inertial frame using a customized feature tracking algorithm. This position is utilized to estimate the velocity of the agents using a first order iterative learning observer. These signals are used in the design of the closed-loop controller. The effectiveness of the controller and observer pair are demonstrated in experiments on spherical magnetic microparticles (see Fig 1).

The major contributions of this work are:

- Deriving a robust closed-loop controller and observer pair for microagents;

- Experimental evaluation of the algorithm using magnetic microparticles.

\section{MODELING MAGNETIC MICROPARTICLES}

In this section, the equations of motion for the spherical microparticles are derived. This is followed by the derivation of the relation between the input currents and magnetic forces used in the electromagnetic actuation.

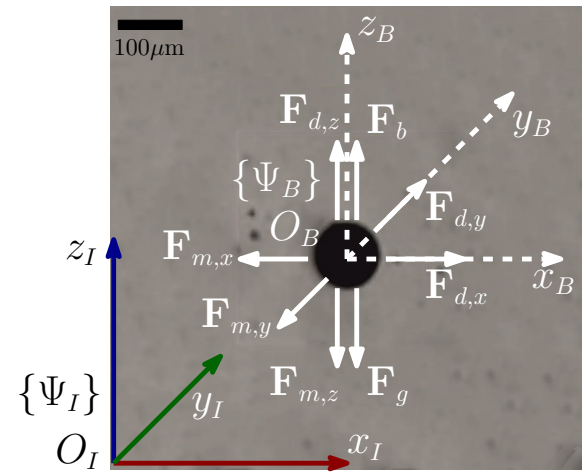

Fig. 2. Graphical representation of the microparticle and the free-body diagram. Here, the frames $\left\{\Psi_{I}\right\}$ and $\left\{\Psi_{B}\right\}$ are related to the inertial and body frames, respectively. For $i \in\{x, y, z\}$, the terms $\mathbf{F}_{d, i}, \mathbf{F}_{m, i}, \mathbf{F}_{b}$ and $\mathbf{F}_{g}$ represent the hydrodynamic drag force, the magnetic force, buoyancy force and the weight of the microparticle, respectively.

\section{A. Equations of motion}

The spherical magnetic microparticles have negligible intrinsic anisotropy. They have an average diameter of 100 $\mu \mathrm{m}$. The details of the kinematic variables and the freebody diagram for the microparticles are shown in Fig. 2. Let $\left\{\Psi_{I}\right\}, O_{I}-x_{I} y_{I} z_{I}$ be an inertial frame with the origin $O_{I}$ and $\left\{\Psi_{B}\right\}$ a body-fixed frame with the origin $O_{B}$ located in the center of mass of the microparticle. The position vector $\mathbf{p}_{B / I}^{I}(t) \in \mathbb{R}^{3}, \mathbf{p}_{B / I}^{I}(t)=\left[\begin{array}{lll}x(t) & y(t) & z(t)\end{array}\right]^{T}$ is the distance from $\left\{\Psi_{I}\right\}$ to $\left\{\Psi_{B}\right\}$ expressed in $\left\{\Psi_{I}\right\}$ and $\mathbf{R}_{B}^{I}(t) \in S O(3)$ describes the orientation of the microparticle with respect to $\left\{\Psi_{I}\right\}$. Here, $t \in \mathbb{R}$ represents the time. In what follows, due to the negligible intrinsic anisotropy assumption and the spherical geometry, we neglect the rotational part in the dynamic equations. The equations of motion of the magnetic microparticles are given as follows:

$$
M_{p} \ddot{\mathbf{p}}_{B / I}^{I}(t)=\mathbf{F}_{d}\left(\dot{\mathbf{p}}_{B / I}^{I}(t)\right)+\mathbf{F}_{m}\left(\mathbf{p}_{B / I}^{I}(t)\right)+\mathbf{F}_{b}+\mathbf{F}_{g}
$$

where $M_{p} \in \mathbb{R}_{>0}$ is the mass of the microparticle. Further, $\mathbf{F}_{d}\left(\dot{\mathbf{p}}_{B / I}^{I}(t)\right) \in \mathbb{R}^{3}, \mathbf{F}_{m}\left(\mathbf{p}_{B / I}^{I}(t)\right) \in \mathbb{R}^{3}, \mathbf{F}_{b} \in \mathbb{R}^{3}$ and $\mathbf{F}_{g} \in \mathbb{R}^{3}$ are the hydrodynamic drag force, the magnetic force, buoyancy force and the weight, respectively. An estimate of the drag force can be computed by considering the geometry of the microparticle, the fluid it is placed in and its maximum velocity. Assuming a maximum velocity of $\max _{t \in \mathbb{R}}\left|\dot{\mathbf{p}}_{B / I}^{I}(t)\right|=1$ $\left[\mathrm{mm} \mathrm{s}^{-1}\right]$ and water as the fluid, a particle Reynolds number of $\operatorname{Re}_{p}<0.1$ can be computed for the microparticle which indicates that it is in the low Reynolds regime. The drag force experienced by a spherical particle moving parallel to direction of the fluid flow for low Reynolds numbers can be computed using Stoke's law as follows:

$$
\mathbf{F}_{d}\left(\dot{\mathbf{p}}_{B / I}^{I}(t)\right)=-6 \pi \eta_{f} r_{p} \dot{\mathbf{p}}_{B / I}^{I}(t)
$$

where $\eta_{f}$ and $r_{p}$ are the dynamic viscosity of the fluid and the radius of the sphere, respectively [20]. The weight and buoyancy forces are given as follows:

$$
\mathbf{F}_{g}=V_{p} \rho_{p} \mathbf{g}, \quad \mathbf{F}_{b}=-V_{p} \rho_{f} \mathbf{g}
$$


where $V_{p}$ and $\mathbf{g}$ are the volume of the microparticle and gravitational acceleration and $\rho_{p}, \rho_{f}$ are the density of the microparticle and fluid, respectively.

\section{B. Magnetic Control Input}

Besides the drag and buoyancy terms derived in the previous section, the magnetic force applied by the electromagnetic coils on the microparticles should be included into the dynamic model [21]. The magnetic flux density for each electromagnet generated at a position $\mathbf{p}^{I} \in \mathbb{R}^{3}, \mathbf{p}^{I}=\left[\begin{array}{lll}p^{I, x} & p^{I, y} & p^{I, z}\end{array}\right]^{T}$ relative to the inertial frame is given by

$$
\mathbf{B}_{i}\left(\mathbf{p}^{I}\right)=\mathbf{R}_{i}^{I} \mathbf{B}_{i}\left(\mathbf{p}^{i}\right), \quad \text { for } \quad i=1, \ldots, n
$$

where $n$ is the number of coils, $\mathbf{R}_{i}^{I} \in S O(3)$ and $\mathbf{B}_{i}\left(\mathbf{p}^{i}\right) \in$ $\mathbb{R}^{3}$ represents the rotation matrix describing the orientation of the $i^{\text {th }}$ coil with respect to the inertial frame, $\Psi_{I}$ and the flux density in its associated local coordinates $\mathbf{p}^{i}=$ $\left[\begin{array}{lll}p^{i, x} & p^{i, y} & p^{i, z}\end{array}\right]^{T}$, respectively [22]. The overall (global) magnetic flux density can be determined by the superposition of the contribution of the $i^{\text {th }}$ electromagnet as [21]

$$
\mathbf{B}\left(\mathbf{p}^{I}\right)=\sum_{i=1}^{n} \mathbf{B}_{i}\left(\mathbf{p}^{I}\right)
$$

with $\mathbf{B}_{i}\left(\mathbf{p}^{I}\right)$ given by (4). The magnetic force $\mathbf{F}_{m} \in \mathbb{R}^{3}$ that the microparticle experiences acting at a point $\mathbf{p}^{I}$ is given by

$$
\mathbf{F}_{m}\left(\mathbf{p}^{I}\right)=\nabla\left(\mathbf{m} \cdot \mathbf{B}\left(\mathbf{p}^{I}\right)\right)
$$

where $\mathbf{m} \in \mathbb{R}^{3}$ and $\mathbf{B}\left(\mathbf{p}^{I}\right) \in \mathbb{R}^{3}$ are the magnetic dipole moment and the global magnetic field given by (5), respectively. It is assumed that in the workspace where the microparticles are controlled, the current varies linearly with the magnetic field which can be expressed as

$$
\mathbf{B}\left(\mathbf{p}^{I}\right)=\sum_{i=1}^{n} \widetilde{\mathbf{B}}_{i}\left(\mathbf{p}^{I}\right) \mathcal{I}_{i}=\widetilde{\mathbf{B}}\left(\mathbf{p}^{I}\right) \mathcal{I}
$$

where $\widetilde{\mathbf{B}}\left(\mathbf{p}^{I}\right) \in \mathbb{R}^{3 \times n}$ is a position-dependent matrix related to the magnetic field evaluated at $\mathbf{p}^{I}$ and $\mathcal{I} \in \mathbb{R}^{n \times 1}$ is the vector of applied currents. The individual elements of matrix $\widetilde{\mathbf{B}}\left(\mathbf{p}^{I}\right)$ depends on the coil parameters such as the number and radius of the windings and the thickness of the coil wires. The particles we consider in this work are superparamagnetic at room temperature, thus the magnetic dipole moment is related the magnetic field as

$$
\mathbf{m}\left(\mathbf{p}^{I}\right)=\frac{\chi_{m}}{\mu_{0}\left(1+\chi_{m}\right)} V_{p} \mathbf{B}\left(\mathbf{p}^{I}\right)
$$

where $\chi_{m}$ and $\mu_{0}$ are the magnetic susceptibility and the vacuum permeability, respectively [5]. Consequently, the forces are related to the currents via the following map:

$$
\begin{aligned}
\mathbf{F}_{m}\left(\mathbf{p}^{I}\right) & =\frac{\chi_{m}}{\mu_{0}\left(1+\chi_{m}\right)} V_{p} \nabla\left(\mathcal{I}^{T} \widetilde{\mathbf{B}}^{T}\left(\mathbf{p}^{I}\right) \widetilde{\mathbf{B}}\left(\mathbf{p}^{I}\right) \mathcal{I}\right) \\
& =\frac{\chi_{m}}{\mu_{0}\left(1+\chi_{m}\right)} V_{p} \mathcal{I}^{T} \frac{\partial}{\partial j}\left(\widetilde{\mathbf{B}}^{T}\left(\mathbf{p}^{I}\right) \widetilde{\mathbf{B}}\left(\mathbf{p}^{I}\right)\right) \mathcal{I} \\
& =\mathbf{h}\left(\mathbf{p}^{I}, \mathcal{I}\right)
\end{aligned}
$$

for $j \in\left\{p^{I, x}, p^{I, y}, p^{I, z}\right\}$. The currents corresponding to the desired magnetic forces are obtained by solving the inverse of the quadratic relation (i.e. $\left.\mathbf{h}^{-1}\left(\mathbf{p}^{I}, \mathcal{I}\right)\right)$ in (11) using the technique described in [23].

\section{DESIGN OF A PRESCRIBED PERFORMANCE CONTROLLER}

In this section, we describe the prescribed performance concept applied to the control of spherical microparticles. This is followed by the design of the prescribed performance controller for the position coordinates of the model derived in Section II. Finally, a sketch of the stability proof is provided.

\section{A. Prescribed Performance Concept}

The prescribed performance concept ensures the convergence of the position error to a predefined and arbitrarily small set with a desired convergence rate and overshoot using decaying functions of time. This is formally expressed as:

$$
\rho_{L}(t)<e(t)<\rho_{U}(t), \quad \forall t \geq 0
$$

where $e(t)$ is a scalar tracking error variable [16]. In (12), the performance functions $\rho_{L}(t)$ and $\rho_{U}(t)$ are sufficiently smooth and bounded functions of time satisfying $\rho_{U}(t)>\rho_{L}(t)$, $\forall t \geq 0$ and $\lim _{t \rightarrow \infty} \rho_{U}(t)>\lim _{t \rightarrow \infty} \rho_{L}(t)$, respectively. For instance, exponential performance functions $\rho_{i}(t)=\left(\rho_{i 0}-\rho_{i \infty}\right) e^{-l_{i} t}+$ $\rho_{i \infty}$ with constants $\rho_{i 0}, \rho_{i \infty}, l_{i}, i \in\{L, U\}$ satisfy the condition (12). The constants $\rho_{L 0}=\rho_{L}(0), \rho_{U 0}=\rho_{U}(0)$ are selected such that $\rho_{L 0}<e(t)<\rho_{U 0}$ holds. The constants $\rho_{L \infty}=\lim _{t \rightarrow \infty} \rho_{L}(t)$ and $\rho_{U \infty}=\lim _{t \rightarrow \infty} \rho_{U}(t)$ are the maximum allowable size of the error $e(t)$ at the steady state. Moreover, the decreasing rate of $\rho_{L}(t), \rho_{U}(t)$ which is affected by the constants $l_{L}, l_{U}$ in this case, introduces a lower bound on the required speed of convergence of $e(t)$. In Section III-B symmetric performance functions are selected for the controller which satisfy $\rho_{L}(t)=-\rho_{U}(t)$.

\section{B. Position Controller}

We utilize the prescribed performance concept to generate magnetic control forces to steer the microparticles to a desired position or to follow a desired trajectory. For this purpose, let us first define the position and velocity errors as

$$
\begin{aligned}
\mathbf{e}_{p}(t) & =\mathbf{p}_{B / I}^{I}(t)-\mathbf{p}_{d}(t) \\
\dot{\mathbf{e}}_{p}(t) & =\dot{\mathbf{p}}_{B / I}^{I}(t)-\dot{\mathbf{p}}_{d}(t)
\end{aligned}
$$

where $\mathbf{e}_{p}(t)=\left[e_{p, x}(t), e_{p, y}(t), e_{p, z}(t)\right]^{T}$ and $\dot{\mathbf{e}}_{p}(t)=$ $\left[\dot{e}_{p, x}(t), \dot{e}_{p, y}(t), \dot{e}_{p, z}(t)\right]^{T}$. The combined position and velocity error can be defined as

$$
s_{p, i}(t)=\dot{e}_{p, i}(t)+\lambda_{p, i} e_{p, i}(t),
$$

for $i \in\{x, y, z\}$ with the gain $\lambda_{p, i} \in \mathbb{R}$ being a positive gain. According to the prescribed performance strategy, using the combined error (15), the magnetic control forces $F_{m, i}\left(\mathbf{p}_{B / I}^{I}(t)\right)$ are selected as

$$
F_{m, i}\left(\mathbf{p}_{B / I}^{I}(t)\right)=-k_{p, i} \ln \left(\frac{1+\frac{s_{p, i}(t)}{\rho_{p, i}(t)}}{1-\frac{s_{p, i}(t)}{\rho_{p, i}(t)}}\right)
$$




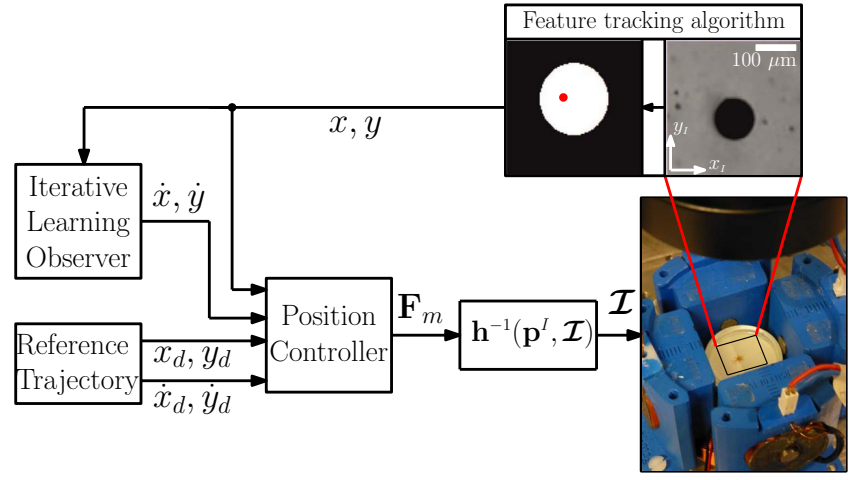

Fig. 3. Closed-loop control system and the cascade controller and observer block diagram. The reference trajectories $x_{d}(t), y_{d}(t)$ and their time derivatives to be followed by the microparticle are used to compute the position (13) and velocity (14) errors. They are used to compute the combined error (15) to obtain the magnetic control forces (16). The forces are converted to currents by solving the inverse relation $\mathbf{h}^{-1}\left(\mathbf{p}^{I}, \boldsymbol{I}\right)$ which are sent to the coils. The time dependence of the signals (e.g. $x(t)$ ) are neglected for brevity.

for $i \in\{x, y, z\}$ where $k_{p, i}>0$ is the control gain. Here, the performance function $\rho_{p, i}(t)$ on the combined position and velocity error (15) is defined as

$$
\rho_{p, i}(t)=\left(\rho_{p, i, 0}-\rho_{p, i, \infty}\right) e^{-l_{p, i} t}+\rho_{p, i, \infty} .
$$

\section{Iterative Learning Observer for Velocity Estimation}

The controller developed in Section III-B makes use of the time derivatives $\dot{x}(t), \dot{y}(t)$ and $\dot{z}(t)$. Different from the configuration variables $x(t), y(t)$ and $z(t)$, these signals cannot be obtained by feedback from the feature tracking algorithm, using microscopic images. Thus, $\dot{x}(t), \dot{y}(t)$ and $\dot{z}(t)$ are estimated using the Iterative Learning Observer (ILO) technique, since numerical differentiation amplifies the noise on $x(t), y(t)$ and $z(t)$ [24]. ILO is selected since it does not require the accurate knowledge of the dynamic equations of the microparticles. Further, it does not make use of the control inputs which depend on the knowledge of the magnetic parameters of the system. The ILO is designed for the $x$ coordinate (Fig. 2) of the microparticle by considering the following system:

$$
\dot{x}(t)=\gamma(t)
$$

where $x(t) \in \mathbb{R}$ is the measurement signal (or the $x$ coordinate) and $\gamma(t) \in \mathbb{R}$ is the unknown time-varying parameter. The ILO for the system (18) is given as

$$
\begin{aligned}
& \dot{\hat{x}}(t)=L_{o}(x(t)-\hat{x}(t))+\hat{\gamma}(t) \\
& \hat{\gamma}(t)=K_{1, o} \hat{\gamma}(t-\tau)+K_{2, o}(x(t)-\hat{x}(t))
\end{aligned}
$$

where $\hat{x}(t) \in \mathbb{R}$ and $\hat{\gamma}(t) \in \mathbb{R}$. Further, $L_{o}, K_{1, o}$ and $K_{2, o}$ are observer gains and $\tau$ is the delay. The ILO for the $y$-coordinate can be derived in a similar way to $x$. The block diagram of the overall control structure is presented in Fig. 3.

\section{Sketch of the Stability Proof}

The following results are used to prove the closed-loop stability of the control system described in Sections III-B and III-C. Consider the initial value problem:

$$
\dot{\xi}=\Phi(t, \xi), \quad \xi(0)=\xi^{0} \in \Omega_{\xi}
$$

with $\Phi: \mathbb{R}_{+} \times \Omega_{\xi} \rightarrow \mathbb{R}^{n}$ where $\Omega_{\xi} \subset \mathbb{R}^{n}$ is a non-empty open set.

Definition 1: A solution $\xi(t)$ of the initial value problem (21) is maximal if it has no proper right extension that is also a solution of (21).

Theorem 1: Consider the initial value problem (21), assume that $\Phi(t, \xi)$ is: a) Locally Lipschitz on $\xi$ for almost all $t \in \mathbb{R}_{+}$, b) piecewise continuous on $t$ for each fixed $\xi \in \Omega_{\xi}$ and c) locally integrable on $t$ for each fixed $\xi \in \Omega_{\xi}$. Then, there exists a maximal solution $\xi(t)$ of (21) on the time interval $\left[0, \tau_{\max }\right)$ with $\tau_{\max }>0$ such that $\xi(t) \in \Omega_{\xi}, \forall t \in\left[0, \tau_{\max }\right)$.

Proposition 1: Assume that the hypothesis of Theorem 1 hold. For a maximal solution of $\xi(t)$ on the time interval $\left[0, \tau_{\max }\right)$ with $\tau_{\max }<\infty$ and for any compact set $\Omega_{\xi}^{\prime} \subset \Omega_{\xi}$ there exists a time instant $t^{\prime} \in\left[0, \tau_{\max }\right)$ such that $\xi\left(t^{\prime}\right) \notin \Omega_{\xi}^{\prime}$.

Theorem 2: Consider the linear system described by

$$
\begin{aligned}
\dot{x}(t) & =A x(t)+B u(t)+F \theta(t) \\
y(t) & =C x(t)
\end{aligned}
$$

and the ILO described by

$$
\begin{aligned}
& \dot{\hat{x}}(t)=A \hat{x}(t)+B u(t)+L(y(t)-\hat{y}(t))+F v(t) \\
& \hat{y}(t)=C \hat{x}(t) \\
& v(t)=K_{1} v(t-\tau)+K_{2}(y(t)-\hat{y}(t))
\end{aligned}
$$

If there exists a positive definite matrix $\mathbf{P}$ satisfying

$$
(A-L C)^{T} P+P(A-L C)=-Q<0
$$

and the ILO parameters, $K_{1}$ and $K_{2}$ are selected such that

$$
0<\alpha K_{1}^{T} K_{1} \leq I, \quad P F=\epsilon\left(K_{2} C\right)^{T} \quad \alpha>\epsilon>1,
$$

then both the state estimation error and the parameter estimation error are bounded.

Considering these preliminary results, the stability of the overall closed-loop control system can be summarized by the following theorem.

\section{Theorem 3: Consider}

i) the system (1),

ii) the initialization set $\Omega_{\xi} \subset \mathbb{R}^{6}$,

iii) the appropriately selected performance functions (17),

The proposed position (16) controllers and the iterative learning observer (19) and (20) solves the prescribed performance stabilization problem stated in Section III-A.

Proof: The stability of the position control loop can be proved following the notions presented in [17]. The proof for the controller using the full-state (position+velocity) information without the observer is done first. This can be done in two steps. First, it can be shown that for the system (15), if the parameters of (15) and (17) are selected as $l_{p, i}<\lambda_{p, i}$, then for each $i$ the error $e_{p, i}(t)$ converges exponentially fast to the residual set $E=\left\{e_{p, i} \in \mathbb{R}:\left|e_{p, i}\right|<\frac{\rho_{p, i, \infty}}{\lambda_{p, i}}\right\} \forall t>0$. In the second step, using Theorem 1 and Proposition 1, it can be shown that the state trajectories of the closed-loop system exists for all future times $(t>0)$ if the initial value of (15) satisfies $\left|s_{p, i}(0)\right|<\rho_{p, i, 0}$. The closed-loop stability with the inclusion of the ILO can be done by utilizing Theorem 2 and input-to-state stability from input $x(t)$ to $\hat{\gamma}(t)$ in (19)-(20). 


\section{EXPERIMENTS}

In this section, we start by briefly introducing our experimental setup. Then, the experimental results related to the prescribed performance controller are introduced.

\section{A. Experimental Setup}

Our experimental setup consists of an array of 6 electromagnetic coils with iron cores placed orthogonally around a fluid reservoir. Four of these coils lie on the same plane to manipulate the planar position of the microagents. The bottom and top coils are not used in the experiments since position information in the $\mathrm{z}$ - direction was not available. Thus, only the position of the microparticles which are floating in the surface can be controlled. For those microparticles, it is assumed that the buoyancy force is balanced by the surface tension force. Each coil is powered by an Elmo 'Whistle' 1/60 DC servo drive (Elmo Motion Control, Petach-Tikva, Israel). A Blackfly 1.4 MP Color GigE PoE (Point Grey Research Inc., Richmond, Canada) camera is mounted on a Mitutoyo FS70 microscope unit (Mitutoyo, Kawasaki, Japan) using a Mitutoyo M Plan Apo 2 / 0.055 Objective. This microscope and camera pair can provide the position information regarding the $\mathrm{x}-$ and $\mathrm{y}$ - directions. We used superparamagnetic microparticles, consisting of iron-oxide in a poly(lactic acid) matrix (PLA Particles-M-redF-plain from Micromod Partikeltechnologie GmbH, Rostock-Warnemuende, Germany). Each individual microparticle have a mass of $M_{p}=7.33 \times 10^{-10}$ $[\mathrm{kg}]$ and a density of $\rho_{p}=1.4 \times 10^{3}\left[\mathrm{~kg} / \mathrm{m}^{3}\right]$. The hydrodynamic parameters of water are $\eta_{f}=1[\mathrm{mPa} \cdot \mathrm{s}]$ and $\rho_{f}=998.2$ $\left[\mathrm{kg} / \mathrm{m}^{3}\right]$, respectively. The parameters regarding the magnetic properties of microparticles and the coils are $\chi_{m}=0.075$, $\mu_{0}=4 \pi \times 10^{7}[\mathrm{~T} \cdot \mathrm{m} / \mathrm{A}], \max \left|\mathbf{B}_{i}\left(\mathbf{p}^{I}\right)\right|<15 \mathrm{mT}$. The details of the image processing algorithm used to detect the center coordinates of the microparticles can be found in [25].

\section{B. Motion Control Results}

We present the results of representative motion control experiments for the prescribed performance controller and Iterative Learning Observer (ILO) detailed in Section III. The controller-observer pair's performance is tested with a constant setpoint obtained by clicking with the mouse on the graphical user interface. The gains of the controller used during the observer are selected as $\lambda_{p, x}=3.25, \lambda_{p, y}=3.25, l_{p, x}=0.5$, $l_{p, y}=0.5$. The gains of the iterative learning observer are selected as $L_{o}=3, K_{1, o}=0.9$, and $K_{2, o}=0.5$. These values are selected considering the actuator limits such as the maximum current and the cut-off frequency of the coils. In all of the experiments we did, the combined error (15) remained within the prescribed performance bounds (17). In the following figures the results of a representative experiment are presented. The recorded setpoint position and the microparticle position obtained by the feature tracking algorithm are shown in Fig. 4. The positions and velocities estimated by the ILO are also shown in Fig. 4. It can be realized that there is negligible overshoot in both $\mathrm{x}$ - and $\mathrm{y}$ - directions and the setpoints are reached in approximately 8 seconds. It can

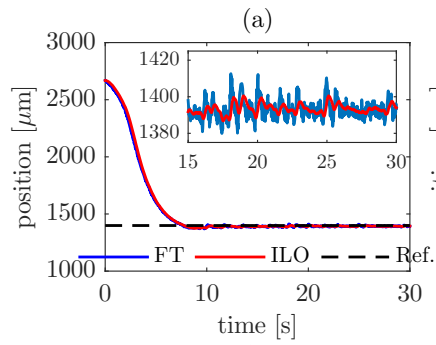

(c)
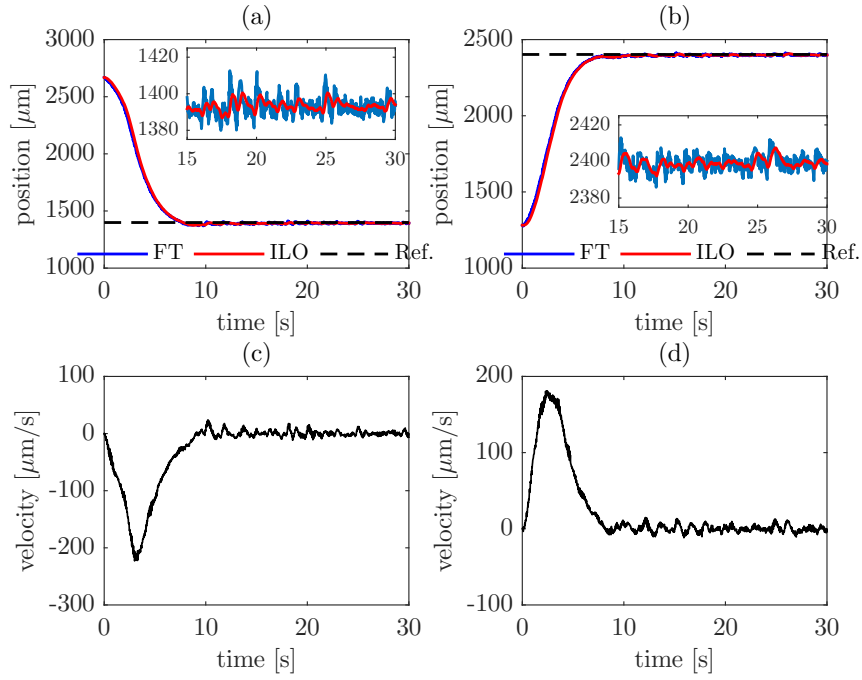

(d)

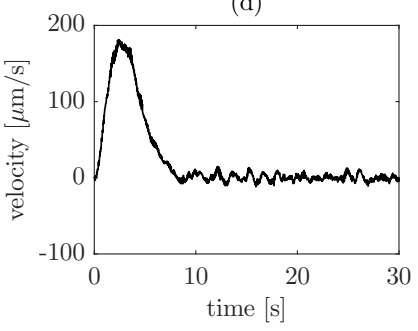

Fig. 4. Results of a representative experiment are shown. The setpoint position (black dashed line), positions estimated by the Iterative Learning Observer (ILO) (red solid line) and the feature tracking algorithm (blue solid line) for $\mathrm{x}$ - and $\mathrm{y}$ - directions are shown in the top plots in (a) and (b), respectively. The velocities estimated by the ILO for $\mathrm{x}$ - and $\mathrm{y}$ - are shown in the bottom plots in (c) and (d), respectively.

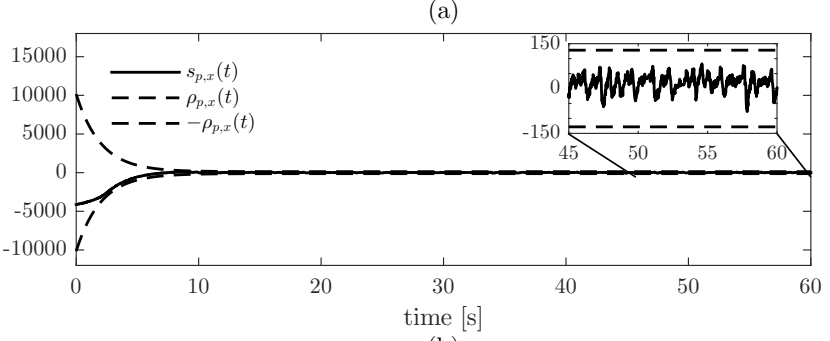

(b)

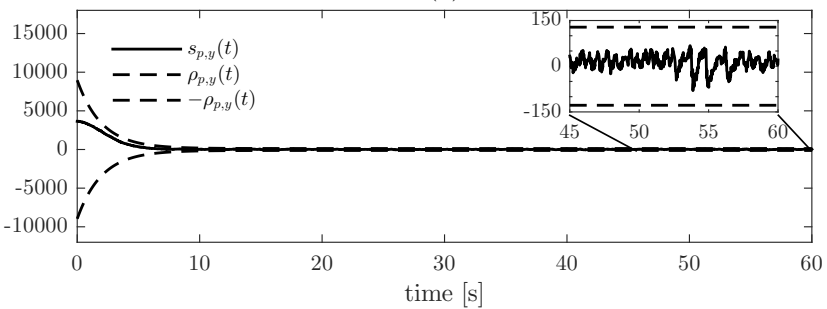

Fig. 5. Results of a representative experiment are shown. The combined position and velocity error (solid line) (15) $s_{p, i}(t)$ for $\mathrm{x}$ - and y-directions are shown in (a) and (b), respectively. The exponentially decaying performance functions $\rho_{p, i}(t)$ are also shown on each plot with dashed lines. The insets show a zoomed version of the aforementioned variables at the steady-state.

also be observed that at steady-state, the standard deviation of the positions estimated with ILO is half of the one obtained using the feature tracking algorithm which indicates that the ILO reduces the noise on the positions. The combined position and velocity error (15) $s_{p, i}(t)$ for $i \in\{x, y\}$ are shown in Fig. 5 . The exponentially decaying performance functions $\rho_{p, i}(t)$ for $i \in\{x, y\}$ are also shown on each plot with dashed lines in Fig. 5. The trace of the microparticle trajectory on the $x-y$ plane is shown in Fig. 6.

\section{CONCLUSIONS AND FUTURE WORK}

We investigated the design of a controller and observer pair for microagents. The prescribed performance concept is 


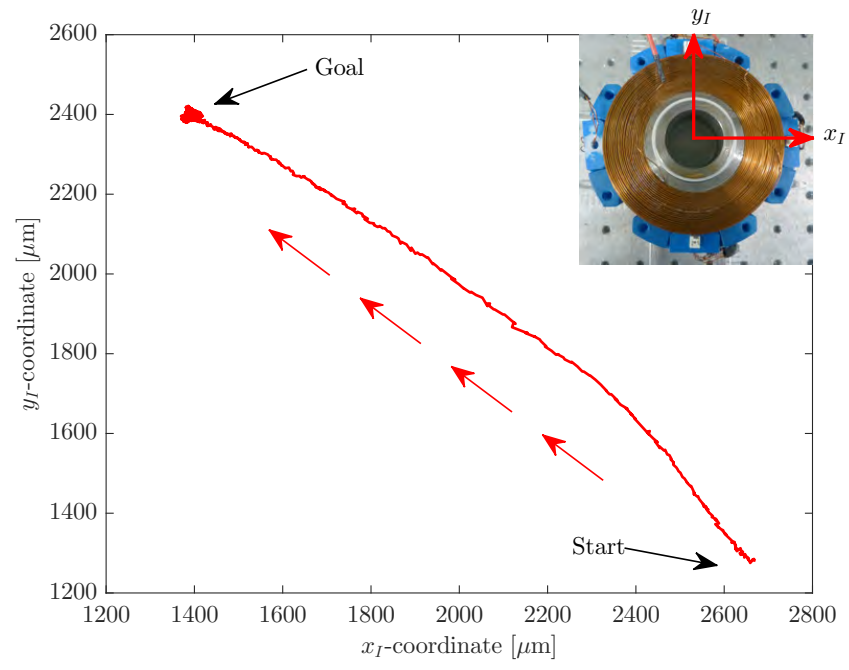

Fig. 6. The trace of $x-y$ trajectory of the microparticle's motion with the initial point $(2668,1275) \mu \mathrm{m}$ and the final setpoint $(1399,2402) \mu \mathrm{m}$. The red arrows next to the actual trajectory of the microparticle represent the direction of motion. The inset in the top right shows the top view of the setup and where the inertial frame is located with respect to it.

utilized to control the position of a microagent to follow a desired trajectory. The velocities required to compute the control action are obtained using an iterative learning observer. Representative experimental results on spherical magnetic microparticles with an average diameter of $100 \mu \mathrm{m}$ are provided which highlight the achievement of the prespecified error metrics such as convergence time and steady-state error. The steady-state errors obtained by the control algorithm are 20 $\mu \mathrm{m}$. The errors converge to the steady-state in approximately 8 seconds.

As part of our future studies, we will investigate the application of this control methodology to different microsized agents including self-propelling ones. The robustness of the control algorithm to environmental disturbances such as fluid flow will also be evaluated. Furthermore, the performance of the control algorithm will also be tested using different imaging modalities.

\section{REFERENCES}

[1] B. J. Nelson, I. K. Kaliakatsos, and J. J. Abbott, "Microrobots for minimally invasive medicine," Annual Review of Biomedical Engineering, vol. 12, no. 1, pp. 55-85, 2010, pMID: 20415589.

[2] M. Sitti, H. Ceylan, W. Hu, J. Giltinan, M. Turan, S. Yim, and E. Diller, "Biomedical applications of untethered mobile milli/microrobots," Proceedings of the IEEE, vol. 103, no. 2, pp. 205-224, Feb 2015.

[3] I. S. M. Khalil, V. Magdanz, S. Sanchez, O. G. Schmidt, and S. Misra, "Biocompatible, accurate, and fully autonomous: a sperm-driven microbio-robot," Journal of Micro-Bio Robotics, vol. 9, no. 3-4, pp. 79-86, 2014.

[4] I. S. M. Khalil, H. C. Dijkslag, L. Abelmann, and S. Misra, "Magnetosperm: A microrobot that navigates using weak magnetic fields," Applied Physics Letters, vol. 104, no. 22, pp. -, 2014.

[5] J. Keuning, J. de Vries, L. Abelmann, and S. Misra, "Image-based magnetic control of paramagnetic microparticles in water," in the Proceedings of the IEEE/RSJ International Conference on Intelligent Robots and Systems (IROS), 2011, pp. 421-426.

[6] K. B. Yesin, K. Vollmers, and B. J. Nelson, "Modeling and control of untethered biomicrorobots in a fluidic environment using electromagnetic fields," The International Journal of Robotics Research, vol. 25, no. 5-6, pp. 527-536, 2006.
[7] L. Zhang, J. J. Abbott, L. Dong, B. E. Kratochvil, D. Bell, and B. J. Nelson, "Artificial bacterial flagella: Fabrication and magnetic control," Applied Physics Letters, vol. 94, no. 6, 2009.

[8] T. Xu, J. Yu, X. Yan, H. Choi, and L. Zhang, "Magnetic actuation based motion control for microrobots: An overview," Micromachines, vol. 6, no. 9 , p. $1346,2015$.

[9] L. Arcese, M. Fruchard, and A. Ferreira, "Adaptive controller and observer for a magnetic microrobot," IEEE Transactions on Robotics, vol. 29, no. 4, pp. 1060-1067, 2013.

[10] H. Marino, C. Bergeles, and B. Nelson, "Robust electromagnetic control of microrobots under force and localization uncertainties," IEEE Transactions on Automation Science and Engineering, vol. 11, no. 1, pp. 310-316, 2014.

[11] K. Belharet, D. Folio, and A. Ferreira, "Control of a magnetic microrobot navigating in microfluidic arterial bifurcations through pulsatile and viscous flow," in the Proceedings of the IEEE/RSJ International Conference on Intelligent Robots and Systems (IROS), 2012, pp. 25592564.

[12] S. Tamaz, R. Gourdeau, A. Chanu, J.-B. Mathieu, and S. Martel, "Real-time mri-based control of a ferromagnetic core for endovascular navigation," IEEE Transactions on Biomedical Engineering, vol. 55, no. 7, pp. 1854-1863, 2008.

[13] J. Choi, S. Jeong, K. Cha, L. Qin, J. Li, J. Park, S. Park, and B. Kim, "Position stabilization of microrobot using pressure signal in pulsating flow of blood vessel," in IEEE Sensors, 2010, pp. 723-726.

[14] C. P. Bechlioulis and G. A. Rovithakis, "A low-complexity global approximation-free control scheme with prescribed performance for unknown pure feedback systems," Automatica, vol. 50, no. 4, pp. 1217 - 1226, 2014.

[15] C. Bechlioulis, M. Liarokapis, and K. Kyriakopoulos, "Robust model free control of robotic manipulators with prescribed transient and steady state performance," in the Proceedings of the IEEE/RSJ International Conference on Intelligent Robots and Systems (IROS), 2014, pp. 41-46.

[16] C. Bechlioulis and K. Kyriakopoulos, "Robust prescribed performance tracking control for unknown underactuated torpedo-like auvs," in the Proceedings of the European Control Conference (ECC), 2013, pp. 4388-4393.

[17] C. Bechlioulis, A. Theodorakopoulos, and G. Rovithakis, "Output feedback stabilization with prescribed performance for uncertain nonlinear systems in canonical form," in the Proceedings of the IEEE $52^{\text {nd }}$ Annual Conference on Decision and Control (CDC), 2013, pp. 5084-5089.

[18] W. Chen and F. N. Chowdhury, "Simultaneous identification of timevarying parameters and estimation of system states using iterative learning observers," International Journal of Systems Science, vol. 38, no. 1, pp. 39-45, 2007.

[19] Y. Kawakami, Y. Eguchi, T. Nimiya, H. Suemitsu, and T. Matsuo, "Velocity and acceleration estimation by iterative learning observer and performance validation with mems-based inertial sensors," International Journal of Advanced Mechatronic Systems, vol. 5, no. 2, pp. 113-121, 2013.

[20] B. R. Munson, T. H. Okiishi, W. W. Huebsch, and A. P. Rothmayer, "Chapter 9 - flow over immersed bodies," in Fundamentals of fluid mechanics. John Wiley \& Sons, Inc., 2013, pp. 480 - 554.

[21] M. Kummer, J. Abbott, B. Kratochvil, R. Borer, A. Sengul, and B. Nelson, "Octomag: An electromagnetic system for 5-dof wireless micromanipulation," IEEE Transactions on Robotics, vol. 26, no. 6, pp. $1006-1017,2010$

[22] S. Miyashita, S. Guitron, M. Ludersdorfer, C. Sung, and D. Rus, "An untethered miniature origami robot that self-folds, walks, swims, and degrades," in the Proceedings of the IEEE International Conference on Robotics and Automation (ICRA), 2015, pp. 1490-1496.

[23] I. Khalil, L. Abelmann, and S. Misra, "Magnetic-based motion control of paramagnetic microparticles with disturbance compensation," IEEE Transactions on Magnetics, vol. 50, no. 10, pp. 1-10, 2014

[24] Y. Kawakami, T. Nimiya, H. Suemitsu, and T. Matsuo, "Performance comparison of velocity estimators with mems-based inertial sensors," in the Proceedings of the International Conference on Advanced Mechatronic Systems (ICAMechS), 2012, pp. 195-200.

[25] A. Sanchez, V. Magdanz, O. G. Schmidt, and S. Misra, "Magnetic control of self-propelled microjets under ultrasound image guidance," in the Proceedings of the $5^{\text {th }}$ IEEE RAS International Conference on Biomedical Robotics and Biomechatronics (EMBS), 2014, pp. 169-174. 